\title{
Trust Breaks Down in Electronic Contexts but Can Be Repaired by Some Initial Face-to-Face Contact
}

\author{
Elena Rocco \\ Collaboratory for Research on Electronic Work (CREW) \\ The School of Information \\ University of Michigan \\ 701 Tappan Street \\ Ann Arbor MI 48109-1234 USA \\ $+13137646715$ \\ rakele@umich.edu
}

\begin{abstract}
Trust is the prerequisite for success when a collaborative task involves risk of individualistic or deceitful behaviors of others. Can trust emerge in electronic contexts? This issue is explored in an experiment in which trust emergence is measured in both face-to-face (F-t-F) and electronic contexts. In this experiment trust is revealed by the degree of cooperation the group is able to reach in solving a social dilemma, i.e. a situation in which advantages for individualistic behavior make group cooperation highly vulnerable. The experiment consists of two stages. The first stage analyzes the cffects of F-t-F and electronic communication on trust. Trust succeeds only with F-t-F communication. The second stage investigates whether a pre-meeting F-t-F can promote trust in electronic contexts. Results are positive. Examination of how people converse in these two contexts sheds some light on the effects of technical characteristics and social circumstances on the emergence of trust.
\end{abstract}

\section{Keywords}

CSCW, trust, cooperation and conflict, teamwork

\section{INTRODUCTION}

Groupware technologies turn out to be not fully appropriate when the prerequisite for action is the establishment of trust. An action demonstrates trust if it 'increases one's vulnerability to another whose behavior is not under control" [1]. Field evidence shows that people engaged in such activities are reluctant to use computer-based media of communication because the lack of face-to-face (F-t-F) contact reduces trust and commitment [2]. Others report need for a prior F-t-F meeting before accepting electronic mail or video-conference as an alternative to F-t-F communication [3]. Sensible as these conclusions are, these studies are based on observations not backed by controlled comparisons.
Reluctance toward electronic media of communication appears to be particularly strong when vulnerability derives from the risk of individualistic or deceitful behaviors. When no standardized procedures or hierarchy guarantee control on individual behaviors, uncertainty and therefore vulnerability increase. In this situation, mutual adjustment and trust are the only coordination mechanisms able to make an organization survive. However, mutual adjustment and trust are very delicate resources rooted in the flow of informal F-t-F relations [4]. This would explain reluctance toward CSCW technologies. As Nohria and Eccles argue, "effectiveness of electronic network will depend on an underlying network of social relationships, based on faceta-face relationships." [5]

A goal of this study is to achieve a better understanding of the requirements for trust, by disentangling causes of vulnerability depending on the task, the characteristics of the groupware technology, and the social circumstances in which the technology is used by a group. The communication technology employed in this study is an electronic mailing list used in a synchronous way. It approached a chat box in terms of speed, but users had to open and close messages like in regular electronic mail. Group members were assigned electronic mail accounts from which they could send messages to the whole group through a group alias. Messages were not anonymous and everyone could read messages posted by everybody else. Messages were accessed like usual electronic mail messages. No private or sub-group exchange of electronic mail was permitted in this experiment. The experiment took place in a laboratory, where six-person groups were engaged in an iterative task involving investment of personal funds and receiving a payment dependent on what others invested. The task embodied a dilemma, known in the literature as "social dilemma" [6]. In a social dilemma individuals face the temptation to defect rather than cooperate to achieve a common goal. Since there is no 
superior control and the incentive to defect is higher than the incentive to cooperate (no matter whether the individual cooperates, she benefits from the outcome of others' cooperation), everybody should theoretically defect, either to take advantage of the situation or to avoid the risk of being the victim of others' opportunism. The moral of a social dilemma is that overall defection leads to miserable results for the group.

But empirical and experimental evidence shows that people naturally ignore the pessimistic moral, in particular when individuals can communicate F-t-F to establish trust within the group $[8,9]$. Positive impact of F-t-F communication in social dilemmas motivates this investigation into whether similar results occur when people communicate electronically by a mailing list.

The study is organized in two stages. The first stage analyzes the effects of F-t-F and electronic communication in groups consisting of strangers. Expectations are that the existence of effects associated with computer-mediated communication will make it difficult for trust to emerge [10]. For instance, de-personalization, lack of contextual presence and non-verbal cues might fail to signal a desire for teamwork and goodwill. The second stage of the study looks at the effect of different social circumstances. In this stage each group meets the day before the experiment to play a game intended to promote group socialization. The day after, the same groups face the usual social dilemma experiment with electronic communication. We expect more cooperation. $F-t-F$ socialization may overcome reluctance toward computer-based technologies of communication when collaborative tasks require trust. On the other hand, it is also possible that future groupware technologies could be designed to include features that are revealed in this study to be necessary to establish trust.

\section{THE EXPERIMENT}

\section{Method}

Experimental groups were composed of six subjects who met for about 3.5 hours to serve in the experiment. Subjects were physically located in the same room; everybody sat in front of a networked computer. Subjects were undergraduate students at the University of Venice (Italy), with experience in the use of electronic mail. Individuals were grouped in such a way that friendship or acquaintance relationships within the group are minimum. Quantitative methods are used to describe groups' performance, participation in the discussion and communication patterns. Qualitative data sources include participant observation, post-experiment questionnaires, analysis of communication transcripts. The experiment is drawn upon a previous experiment for the study of F-t-F communication on the emergence of cooperation [9].

\section{Experimental Task}

The individual task consisted of an investment in a market. The same task was repeated for about 28 rounds. In each round, once everybody invested, the group payoff was

\begin{tabular}{|l|c|}
\hline $\begin{array}{l}\text { Amount given to an } \\
\text { individual to invest per } \\
\text { round }\end{array}$ & 13 tokens \\
\hline Number of subjects & 6 \\
\hline $\begin{array}{l}\text { group investment at social } \\
\text { optimum (cooperation) }\end{array}$ & 36 tokens \\
\hline $\begin{array}{l}\text { group investment at naive } \\
\text { social optimum } \\
\text { (cooperation) }\end{array}$ & 46 tokens \\
\hline $\begin{array}{l}\text { group investment at } \\
\text { individualistic optimum } \\
\text { (non cooperation) }\end{array}$ & 60 tokens \\
\hline $\begin{array}{l}\text { Individual payoff at social } \\
\text { optimum }\end{array}$ & 119 cents \\
\hline $\begin{array}{l}\text { Individual payoff at } \\
\text { individualistic optimum }\end{array}$ & 95 cents \\
\hline $\begin{array}{l}\text { Individual payoff for 1 non } \\
\text { cooperator when only } 5 \\
\text { subjects respect the social } \\
\text { optimum }\end{array}$ & 159 cents \\
\hline
\end{tabular}

Table 1. Some values of the experiment

calculated in correspondence to the sum of individual investments. Then each subject received her individual payoff as a share of the group payoff. Subjects had a table with complete information about the market payoff in correspondence of each possible investment. At the beginning of each round each subject received the same number of tokens to invest. She also received an updated record of her past individual investments and payoffs, and a record of the total group investment in each round of the game (investments are measured in tokens, payoffs are calculated in cents). But she was not given information about how much each person invested. Thus a subject could monitor group behavior but not individual ones.

At the end of the experiment everybody received a monetary payoff proportional to the individual payoff accumulated in the game.

The main problem faced by the subjects was that the market hid a trap: after a certain level of group investment, the bigger the group investment, the lower the group payoff. In other words, the market payoff had a parabolic profile: if subjects made large investments everybody was worse off. People however wanted to make large investments because the way in which the individual share of group payoff was calculated in the game. This share was proportional to the individual investment of the group investment. For instance, if a subject invested 10 tokens and the group had invested 40 tokens, then this subject got $10 / 40$ of the group payoff. Thus, this rule encouraged everybody to make large individual investments. The dilemma was: should I make small investments to avoid the trap built in the market or large investments to earn as much as possible? Cooperation or defection? When a subject chose a small investment he was aware of being vulnerable, since there was no guarantee that others would have done the same. Total group investment was the only signal every participant 
received at the end of each round to measure group cooperation.

Two group investments are particularly relevant: the social optimum indicating the optimal amount a cooperative group as a whole should invest, and individualistic optimum indicating the optimal amount for a group of noncooperative people. Social optimum provides higher payoff to everybody but is more risky, since a single defector can earn a lot exploiting the small investment of the others (Table 1). Subjects had all the necessary information to calculate these solutions. Miscalculation of the social optimum brings to a result called naïve social optimum. It indicates that subjects are cooperating but without perfectly optimizing how much they can earn from the market.

In this study, after the $10^{\text {th }}, 15^{\text {th }}$ and $20^{\text {th }}$ rounds of this game, the group members participated in a round table-like discussion. The purpose of the discussion was to reach an agreement to establish cooperation. Agreements were not enforceable. Indicating with $\mathrm{X}$ a round of the task and with $C$ a communication period, the experiment has the following structure:

\section{XXXXXXXXX C XXXXX CXXXXX CXXXX....}

\section{Communication conditions}

Twelve groups participated in the experiment. Different communication conditions were designed for them in the 3 communication periods. In the first stage of the study 3 groups communicated F-t-F and 6 groups used a non anonymous electronic mailing list. In the second stage 3 groups played a F-t-F game the day before the experiment and then participated in the experiment with communication supported by a non-anonymous electronic mailing list.

\section{Condition l: Face-to-Face}

Subjects had the possibility to discuss for 10 minutes in each communication period. They left the computer and met around a table in another room. A hidden camera recorded groups' behavior for the analysis of communication and patterns of actions.

\section{Condition II: Electronic Mailing List}

Subjects had the possibility to discuss for 30 minutes in each communication period. Communication was supported by an electronic mailing list resembling important features of a traditional F-t-F round table: messages were not anonymous and everybody could read everybody else's messages. Messages were recorded in a file for the analysis of communication protocols.

Condition III: Face-to-Face socialization before using the electronic mailing list

This condition differed from the previous one to the extent that subjects were exposed to a special treatment the day before the experiment. The treatment consisted of playing a 45-minute game aimed to elicit socialization through ingroup collaboration. In this game the three groups competed against each other to build the best tower out of paper according to some pre-defined criteria. The day after the same groups played the usual experiment with communication supported by a mailing list.

\section{FIRST STAGE OF THE STUDY}

In the first stage, conditions I and II are analyzed. The study aimed to see if trust breaks down when electronic communication replaces $\mathrm{F}-\mathrm{t}-\mathrm{F}$ communication and individuals face a task characterized by high degree of vulnerability.

\section{Results}

Some recurring aspects describe groups' behavior in condition I. First, after the first communication period stable cooperation emerged. Second, at the end of each communication period groups reached a unanimous agreement even if some subjects show skepticism about the optimality of the decision. Third, groups got always locked in a naive social optimum during the first communication period. Only two groups were capable of finding the social optimum and implemented the right strategy after the second communication period. On average group investment coalesced at a low level (Fig. 1). This indicates that subjects had overcome the vulnerability intrinsic in the task and had instead established trust-based cooperation.

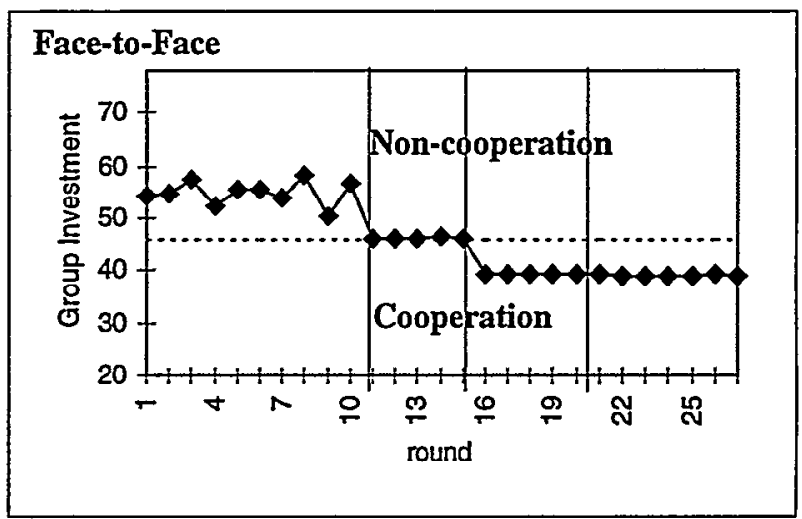

Fig 1.

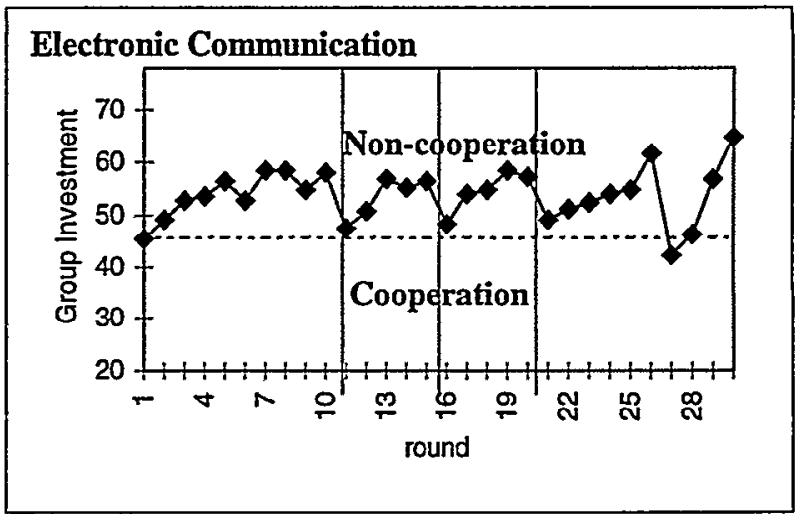

Fig. 2. Average group investment in the rounds of the experiment. Vertical lines represent the communication periods. The horizontal line indicates the threshold for cooperation: above 46 (naïve social optimum) non cooperation occurs. 
In condition II individuals showed less stable and highly individualistic behavior than in condition $I$, reflected in the profile of the group investment (Fig. 2). Also in this condition some recurring aspects characterize group behavior. First, cooperation never succeeded despite of several attempts to define an agreement during the communication periods ( 15 out of 18 communication periods end up with a verbal agreement). Agreements were rarely unanimous (5 out of 15 agreements reach unanimity). However every agreement was systematically disregarded: immediately after communication, a few subjects broke the covenant, triggering a snowball-like defection of the rest of the group. In Fig. 2 notice that group investment always gradually rose after a communication period. The deceitful behavior of early defectors is a concrete example of opportunism, here referred to as "electronic opportunism" to emphasize that interactions occurred electronically. Electronic opportunism was highly profitable for early defectors who made large investments taking advantage of smaller investors.

The second commonality is that communication had a decreasing impact on eliciting cooperation because individuals experienced disenchantment and frustration about the group's trustworthiness. Frustration increased also because the flow of communication got very chaotic as time passed.

Third, groups were better in problem solving than in condition $I$ in terms of number and quality of solutions generated. In fact groups communicating by mailing list generated and explored more solutions ( 5 in condition II versus 3.4 in condition I on average, per communication period). Also, 4 out of 6 groups did not get locked in the naive social optimum during the first communication period. However, in condition II only 2 groups were able to deploy the social optimum solution in a non unanimous agreement. Lack of trust and skepticism weakened individual intentions to respect any agreement. On average group investment was much higher than in condition I, indicating the breakdown of trust in the groups.

\section{Interpretation}

Analysis of video tapes and communication protocols revealed some factors that could be responsible for the differences observed between the two communication conditions.

Inielligible communication as a prerequisite for cooperation. Participation in the communication periods was very different when subjects used an electronic mailing list. This technology does not provide support for rules of sequentiality and turn taking that govern F-t-F communication [11]. Sequentiality permits the discussion to gain coherence. In the mailing list participants received a multitude of messages whose topics were rarely in sequence. In contrast to F-t-F communication in which speakers govern the sequence of the discussion, in the mailing list sequence depends on the recipient of the messages who decides what to read first. The lack of need to take turns permits several threads to be discussed at the same time, causing the focus of the discussion to be lost. But it also meant that one can avoid responding to questions and ignore messages because of the lack of physical presence which usually compels responses. The breakdown of traditional rules of communication makes discussion nearly unintelligible. The argument here is that absence of cooperation in achieving an intelligible discussion fed skepticism about the possibility of achieving cooperation on the task.

\section{"Cheap" coordination facilitates cooperation.}

Cheap coordination refers to mechanisms such as informal division of labor and leadership that often takes place in Ft-F settings. In condition I a cheap division of labor characterized group problem solving. Analysis of video tapes revealed that different sub-groups were engaged in different activities: some did calculations, others explored alternative solutions, others explained the current strategy to those who showed skepticism or doubts about the strategy to adopt. The decomposition into sub-groups emerged by evolution rather than by design [12]. In condition II this coordination never emerged. This explains why subjects came up with the solution individually, exploring solutions and doing calculations alone. Ultimately, individual problem solving made it harder to reach an agreement because everybody wanted to impose her or his own solution onto the others.

Moreover, informal leadership of one or two subjects emerged in condition I. Leadership is related to the effect known as "first advocacy effect" [13]. First advocacy effect says that the first advocate is likely to gain leadership attribution by group members. That person's proposal is more likely to be implemented as group solution. Analysis of communication protocols shows that in condition I the first solution was indeed generally deployed in a group agreement. In condition II first advocacy effect was almost absent. Many solutions were simultaneously discussed until the end of the communication period. Then subjects engaged in a confused last minute voting that made it very hard to define the agreement.

Early establishment of social norms shapes cooperative attitudes.

Norms and conventions represent a crucial aspect of the groups' life. In both conditions I and II subjects engaged in the definition of norms. However, norms assumed very different connotations. In condition I groups stressed from the early minutes of the first communication period the need to respect cooperation, even in the face of possible defection. Conversely, in condition II groups postponed the discussion of norms to the end of the first communication period. Bad timing of social norms establishment might have increased the sense of vulnerability. At the end of the communication period subjects experienced high anxiety because they wanted to reach some agreement. The need 
for cooperation was enforced by threats of defection as a deterrence to punish possible opportunism (a variation of the famous tit-for-tat). In condition I the subjects never threatened. After the first communication period they did not need to reassert social norms once cooperation emerged. In condition II, the existence of electronic opportunism enhanced the need to establish norms. Aggressive expressions of disappointment (flaming), mistrust and blame dominated the second and third communication periods. The norm of cooperation was not the only norm to be violated.

\section{SECOND STAGE OF THE STUDY}

The previous results indicate that F-t-F communication elicits trust when the group is engaged in a task characterized by high degrees of vulnerability. However, when the group's relationships already carry a sort of group identity we could expect that cooperation might more easily emerge even if communication occurs electronically. Group identity, common values and history are recognized as prerequisites for long lasting trust-based cooperation [8]. Thus social circumstances might have a large impact on the use of a computer-based medium of communication. The second stage of the study investigated this hypothesis by introducing F-t-F socialization before groups engaged in electronic communication.

\section{Results}

In condition III, subjects demonstrated firm willingness to come to an agreement from the first communication period in marked contrast to subjects in condition II. But cooperation initially fell as indicated by the group investment that increased between the first and second communication periods (Fig. 3). Analysis of communication protocols suggests that cooperation fell not because of electronic opportunism, but because the groups experienced difficulties in understanding the final agreement. In one case, for instance, the group split in two sub-groups, each cooperating, but using different agreements. After the second communication period all groups overcame communication problems and reached stable cooperation. On average, the second and third communication periods lasted respectively 18 and 5 minutes, in spite of having 30 minutes available, since participants quickly converged to the agreement and asked the experimenter to continue with more investment rounds.

A post-experiment questionnaire confirmed that the F-t-F treatment had successfully achieved some degree of socialization. On average 4.3 subjects/group reported that it stimulated group identity and 3.6 of them thought the rest of the group experienced the same feeling. Also 4.6 subjects/group reported that prior F-t-F positively affected cooperation during the experiment.

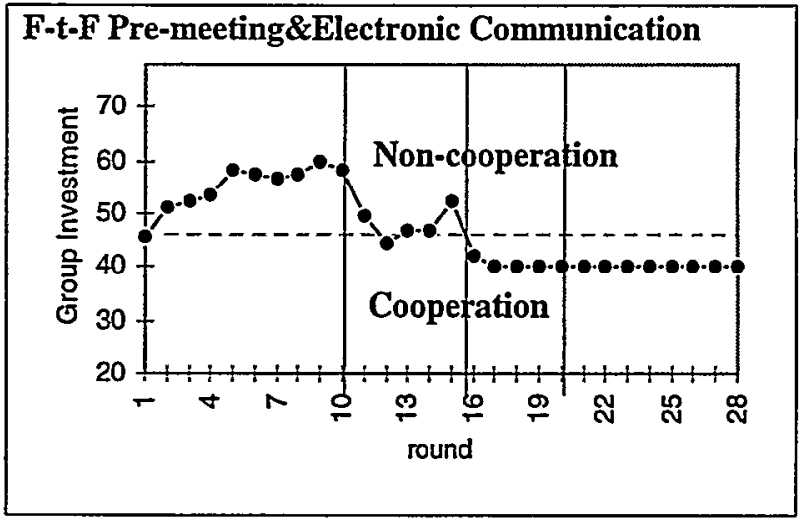

Fig. 3. Average group investment in the rounds of the experiment. Vertical lines represent the communication periods. The horizontal line indicates the threshold for cooperation: above 46 (naïve social optimum) non cooperation occurs.

\section{Interpretation}

Results are highly informative about the possible effects of F-t-F socialization before a text-based technology, like an electronic mailing list, is used for communication. Groups that had established socialization and perhaps trust were able and willing to overcome difficulties due to the communication technology. Some observations can be made about factors that make trust successful in condition III.

Group identity affects task's interpretation.

The content analysis of messages exchanged by the mailing list indicated that groups recognized a sort of group identity. Behaviors such as answering each others' questions, frequent use of the pronoun "us" rather than "anyone" or "you", and emphasis given to the concept of "our interest" were very common. This finding contrasts what was noticed in condition II, where subjects' behaviors resembled that of competitors in a market rather than that of members of a group. Frequent use of "anyone" and "you", emphasis on "my payoff" versus "your payoff" and statements such as "loyalty is not a requirement" conveyed the absence of group identity in condition II. The argument here is that the existence of group identity affects the task's interpretation to the extent that the same task can assume different natures according to the social circumstances. In condition III subjects stated that "the game's goal is to maximize group payoff" and "our task is to figure out how to reach even individual payoffs". Conversely, in condition II individuals framed the task in individualistic terms, trying to maximize their own payoff, with the awareness that " $a$ cartel will be difficult to sustain."

Cooperation revealed by styles of use of the technology. Groups in condition III appropriated the technology differently from groups in setting II. In setting II discussion was dominated by many parallel threads, causing chaos and ambiguity. As a consequence, everybody was discouraged to read and understand the discussion. Many threads were 
alive until the last minute of the communication period. This boosted disagreement rather than convergence toward a consensus. Some threads invoked cooperation. But others seriously undermined cooperation proposing sabotages and stating the impossibility of reaching consensus. Conversely, a lower number of threads that decreased over time characterized communication in condition III. Individuals cooperated to make the discussion more intelligible. This facilitated the convergence toward the agreement.

\section{Fespect of social norms signals trustworthiness.}

Like in condition I, subjects showed the need to establish social norms from the first minute of communication. They sent messages like "the group must cooperate". Then cooperation was taken for granted once the agreement was reached. There was no need to enforce the agreement by threats. Also, in condition III subjects never showed disappointment or frustration about the difficulties in using an electronic mailing list, nor expressed anger for the initial failure of cooperation. This behavior contributed to diffusing confidence about cooperation. Subjects showed concerns for the norm of fairness, especially after the initial breakdown of cooperation. This behavior preserved the group from dissolution. Instead, in condition II breakdown of cooperation and electronic opportunism created the breakdown of social norms, provoking threats, flaming and bluffs (misrepresentation of the purpose of past investments, phony declarations of honesty, etc).

\section{CONCLUSION}

A potential source of teamwork's failure is the perception of vulnerability due to the risk of individualistic or deceitful behaviors of team members. In these cases establishment of trust is the prerequisite for action. The greater the risk, the greater the need for trusting relationships because people do not take great risks with those they do not trust. Communication plays a central role in forging trusting relationships. However, when communication occurs electronically, in particular in text-based formats, the perception of vulnerability can increase. As a consequence, team members fail to develop trust.

This study sheds some light on the impact of electronic versus $F$-t-F communication on trust development, with a specific focus on the issue of trust in teams of strangers. Two main conclusions can be drawn. The first stage of the study reveals that factors such as intelligible communication, cheap coordination and early establishment of collaborative norms are mainly responsible for the successful cmergence of trust in F-t-F context. When communication is supported by an electronic mailing list these factors appear fragile or even absent. This causes the breakdown of trust and, ultimately, of cooperation. Therefore, a first conclusion is that electronic communication might turn out to be inappropriate to support teamwork when trustworthiness is a prerequisite for action and members cannot rely on past experiences shared with the others. The need to verify whether the technology is the only cause responsible for the breakdown of trust led to the second stage of the study in which F-t-F socialization is introduced before individuals use the electronic mailing list. The study shows that trust can be achieved when team members have an initial F-t-F contact. Various elements signal that trust is in place: existence of group identity, style of use of the technology, respect of social norms.

Several research avenues could follow up these results. From the technical standpoint, design of future communication technologies should take into account the role of the factors revealed to be necessary for the development of trust. For instance, the study emphasizes the importance of intelligibility of electronic communication. Intelligible communication reduces risks of misunderstandings and encourages participation. Applications more advanced and more structured than mailing lists could support better intelligibility. Other tools could be developed to increase intelligibility by visual aids (e.g. visualization of communication threads, with messages displayed along the line in chronological order and situated at different levels to indicate the relative importance). Visualization of the communication threads might improve intelligibility and reduce the rigidity implicit in text-based forms of communication. Other features could be added depending on the possible causes of vulnerability intrinsic in the task (e.g. need for visual contact).

A different question regards the appropriate use of actual group technologies when a team's members need to establish trust before engaging in collaborative activities. It might be that reluctance toward groupware could indicate lack in the group trustworthiness or reliability. Although collaborative activities do not always imply risks of opportunistic or deceitful behavior like in a social dilemma described above, social norms play a crucial role in coordinating group behavior. For instance, a group coping with time pressure may need social norms to deal with exceptional events (occasional delays, unavailability of a team member, ...). To avoid risks of delays, uncertainties or internal conflicts, social norms should be discussed beforehand.

Socialization facilitates definition of social norms in many ways. After establishing personal relationships, individuals are usually more comfortable in revealing personal opinions, preferences and concerns [7]. Such disclosure brings to the surface potential obstacles, making it easier to foresee difficulties and negotiate appropriate social norms. Moreover, socialization supports the establishment of the group identity and the existence of group identity enhances the respect of norms [9]. As a consequence computersupported collaboration could be improved by planning ahead one or more face-to-face meetings to support socialization. In general, this study suggests the need to explore further the issue of socialization in electronic 
environments. We need to analyze what the impediments to socialization are when people rely on computer-based media of communication. Also, we need to investigate whether a different or more intensive use of these media could overcome these impediments. The success of CSCW and design of future applications rely on our understanding of the transient and permanent effects of technology on teamwork.

\section{ACKNOWLEDGMENTS}

I am grateful to Judy Olson for her comments on and support with this research. I also would like to thank the members of the Collaboratory for Research on Electronic Work for their insightful feedback on a previous draft of this paper.

\section{REFERENCES}

1. Zand, D. E. Trust and Managerial Problem Soving. Administrative Science Quarterly, 1972, 17, 229-239.

2. Olson, J. S. and Teasley, S. Groupware in the Wild. Lessons Learned from a Year of Virtual Collocation. In Proceedings of CSCW 96 (Boston, Nov. 1996), ACM Press, 419-427.

3. O'Hare-Devereaux, M. and Johansen, R. Globalwork: bridging distances, culture and time. San Francisco, Jossey-Bass Publisher, 1994.

4. Mintzberg, H. The Structuring of Organizations. Prentice Hall, Englewood Cliffs, 1979.

\footnotetext{
Permission to make digitalhard copies of all or part of this material for personal or classroom use is granted without fee provided that the copies are not made or distributed for profit or commercial advantage, the copyright notice, the title of the publication and its date appear, and notice is given that copyright is by permission of the ACM, Inc. To copy otherwise, to republish, to post on servers or to redistribute to lists, requires specific permission and/or fee.

CHI 98 Los Angeles CA USA

Copyright 1998 0-89791-975-0/98/4..\$5.00
}

5. Nohria, $N$ and Eccles, R. Face-to-Face Making Network Organization Work. Nohria, N. and Eccles, R. (eds.) Networks and Organizations. Cambridge, MA, HBS Press, 228-308, 1992.

6. Dawes, R. M. Social Dilemmas. Annual Review of Psychology, 1980, 31, 169-193.

7. Pruitt, D.G. Negotiation Behavior. New York: Academic Press, 1981.

8. Sally, D. Conversation and Cooperation in Social Dilemma. Rationality and Society, 1995, 7(1), 58-92.

9. Ostrom, E., Walker, J. and Gardner, R. Covenants with and without a Sword: Self-Governance is Possible. American Political Science Review, 1992, 86, 404-417.

10.Garton, L. and Wellman, B. Social Impacts of Electronic Mail in Organizations: A Review of the Research Literature. Brant R. Burleson (Ed) Communication Yearbook (18), Thousand Oaks, CA: Sage, 1995.

11.Suchman, L. A. Plans and Situated Action. The problem of human machine communication. Cambridge University Press, NY, 1987.

12.Hutchins, E. Organizing Work by Adaptation. Organization Science, 1991, 2(1), 14-39.

13. Weisband, S. P. Group Discussion and First Advocacy Effect in Computer-Medited and Face-to-Face Decision Making Groups. Organizational Behavior and Human Decision Processes, 1992, 53, 352-380. 